\title{
THE FACTORIZATION AND REPRESENTATION OF LATTICES
}

\author{
BY \\ GEORGE MARKOWSKY( $\left.{ }^{1}\right)$
}

\begin{abstract}
For a complete lattice $L$, in which every element is a join of completely join-irreducibles and a meet of completely meet-irreducibles (we say $L$ is a jm-lattice) we define the poset of irreducibles $P(L)$ to be the poset (of height one) $J(L) \cup M(L)(J(L)$ is the set of completely join-irreducibles and $M(L)$ is the set of completely meet-irreducibles) ordered as follows: $a<_{P(L)} b$ if and only if $a \in J(L), b \in M(L)$, and $a \nless L^{b}$. For a jm-lattice $L$, the automorphism groups of $L$ and $P(L)$ are isomorphic, $L$ can be reconstructed from $P(L)$, and the irreducible factorization of $L$ can be gotten from the components of $P(L)$. In fact, we can give a simple characterization of the center of a jm-lattice in terms of its separators (or unions of connected components of $P(L)$ ). Thus $P(L)$ extends many of the properties of the poset of join-irreducibles of a finite distributive lattice to the class of all jm-lattices.

We characterize those posets of height 1 which are $P(L)$ for some jmlattice $L$. We also characterize those posets of height 1 which are $P(L)$ for a completely distributive jm-lattice, as well as those posets which are $P(L)$ for some geometric lattice $L$.

More generally, if $L$ is a complete lattice, many of the above arguments apply if we use "join-spanning" and "meet-spanning" subsets of $L$, instead of $J(L)$ and $M(L)$. If $L$ is an arbitrary lattice, the same arguments apply to "join-generating" and "meet-generating" subsets of $L$.
\end{abstract}

This paper concerns the problem of representing lattices by means of closure operators on partially ordered sets of height 1 . Every relation between two sets $R \leqslant X \times Y$ induces a Galois connection between the power set of $X$ and the power set of $Y$, and hence determines a lattice $L(R)$ of closed sets (in $X$, say). If $L$ is a finite lattice, and $X$ and $Y$ are the sets of join- and meetirreducible elements of $L$, and $R$ is the relation $\$$, then $L \cong L(R)$. This idea extends trivially to complete infinite lattices in which every element is a

Received by the editors August 17, 1973 and, in revised form, January 15, 1974. AMS (MOS) subject classifications (1970). Primary 06A15, 06A20, 06A23, 06A35; Secondary 06A30, 06A45.

Key words and phrases. Poset of irreducibles, completely join-irreducible, Galois connection, irreducible factorization, representations, group of automorphism, geometric lattice, poset of join-irreducibles, join-spanning set, distributive lattice, separators, center.

(1) The results described here are partly contained in the author's doctoral thesis [5] which was partly supported by ONR Contract N00014-67-A-0298-0015. 
join of completely join-irreducibles and a meet of completely meet-irreducibles (which we have called jm-lattices). We call $R$ the poset of irreducibles and denote it by $P(L)$. More generally, if $L$ is complete, the same arguments apply if $X$ and $Y$ are "join-spanning" and "meet-spanning" subsets of $L$. If $L$ is an arbitrary lattice, the same arguments apply if $X$ and $Y$ are "join-generating" and "meet-generating" subsets of $L$. If $X$ and $Y$ are invariant under all automorphisms of $L$, then in all three cases the automorphism group of the bipartite directed graph associated with $R$ is isomorphic to the automorphism group of $L$.

In this paper, we examine how properties of $L$ can be described in terms of properties of the relation $R$. The main object is to characterize those relations which arise from certain kinds of lattices.

Not every relation comes from a lattice, and $\$ 1$ concerns the problem of describing those which do. We consider this problem for each of three possible choices: $L$ a jm-lattice, $L$ a complete lattice, $L$ an arbitrary lattice. Certain of the results can be expressed conveniently in the language of topological spaces.

In $\S 2$, we characterize the relations arising from completely distributive jm-lattices (which are isomorphic to lattices of order ideals of some partially ordered set). In this case, the relation $R$ provides an alternative to the wellknown "poset of join-irreducibles," and shares many of its properties.

In $\S 3$, we do the same for geometric lattices. By applying this condition and its dual, we obtain a characterization of complemented modular lattices of finite length.

In $\S 4$, we show how the direct factorizations of a jm-lattice $L$ can be derived immediately from the poset of irreducibles of $L$. In particular, $L$ is directly irreducible if and only if $P(L)$ is connected (as a graph). This leads to an elementary description of the center of a lattice in terms of its separators (or unions of connected components of $P(L)$ ).

Finally, in $\S 5$ we give a number of examples which illustrate the material presented in the earlier sections.

The poset of join-irreducible elements $P$ of a finite distributive lattice $L$ contains $L$ in a coded form, since $L$ can be reconstructed from $P$. Furthermore, the automorphism group of $P$ is isomorphic to the automorphism group of $L$, and the direct factorization of $L$ can be gotten directly from the connected components of $P$. However, $P$ is naturally isomorphic to the poset of meet-irreducible elements of $L$, i.e., the meet-irreducible elements do not contain any information about $L$ not already contained in $P$. Thus one might suspect that in the nondistributive case one would need to include both the joinirreducible and meet-irreducible elements of a lattice in any representation of it which would have the same properties as the poset of join-irreducibles of a finite 
distributive lattice. The results in this paper show that such a representation can be found, and that we only need to add the meet-irreducible elements to the join-irreducible elements.

1. Basic results. Let $X$ be a set; by $|X|$ we shall denote the cardinality of $X$ and by $2^{X}$ we shall denote the power set of $X$. Let $n$ be a nonnegative integer; by $\underline{n}$ we shall mean the set $\{1, \cdots, n\}$. Thus $\underline{0}=\varnothing$. Note that $2^{n} \neq 2^{n}$. We use $\cup$ for disjoint union. We will usually write $\leqslant$ for set inclusion and $<$ for proper set inclusion. If $X$ is an object with some sort of structure, by $\operatorname{Aut}(X)$ we shall mean the set of all automorphisms of $X$, i.e., bijections which preserve the given structure. We shall use $O(I)$ to denote the least (greatest) element of a lattice $L$ if it exists. As usual $O(I)$ is the empty join (meet).

Definition 1. Let $L$ be a lattice and $X \leqslant L$.

(a) We say that $X$ join-spans (meet-spans) $L$ if $0 \notin X(I \notin X)$ and for every $w \in L$ there exists $X_{w} \leqslant X$ such that $w=\sup X_{w}\left(w=\inf X_{w}\right)$. Note $L$ join-spans (meet-spans) itself. We will use this $X_{w}$ notation throughout.

(b) We say that $X$ join-generates (meet-generates) $L$ if for every $w \in L$ there exists a finite nonempty subset $X_{w}$ of $X$ such that $w=\sup w(w=$ $\inf X_{w}$ ). Note that $L$ join-generates (meet-generates) itself. We will use this $X_{w}$ notation throughout.

(c) If $L$ is a jm-lattice, by $J(L)(M(L))$ we denote the set of all completely join-irreducible (completely meet-irreducible) elements of $L$. Clearly; $J(L)$ $(M(L))$ join-spans (meet-spans) $L$.

REMARK. We note that any lattice with no infinite chains is a jm-lattice. More generally, any lattice such that it and its dual are both compactly generated is a jm-lattice (see $[3, \mathrm{p} .43$, Theorem 6.1]).

Definition 2. Let $P$ be a poset. By a link in $P$, we mean a finite sequence of elements of $P$, say $a_{1}, \cdots, a_{m}$, such that, for $i \in \underline{m-1}, a_{i} \leqslant$ $a_{i+1}$ or $a_{i} \geqslant a_{i+1}$. We introduce an equivalence relation of $P$. Let $x, y \in P$; we say that $x \sim y$ if there exists a link $a_{1}, \cdots, a_{m}$ such that $a_{1}=x$ and $a_{m}=y$. We define the components of $P$ to be the equivalence classes of $P$ with respect to $\sim$. A poset is connected if it has exactly one component.

DEFinition 3. (a) By a bipartite digraph $D$, we mean a triple $(X, Y, A)$, where $X$ and $Y$ are sets, $X \cap Y=\varnothing$, and $A \leqslant X \times Y . A$ is called the set of arcs. If $S \leqslant X$, by $\mathrm{Ou}(S)$ we mean $\{y \in Y \mid$ there exists $x \in S$ such that $(x, y) \in A\}$. Similarly, if $T \leqslant Y$, by $\operatorname{In}(T)$ we mean $\{x \in X \mid$ there exists $y \in T$ such that $(x, y) \in A\}$. If $x \in X[y \in Y]$ we write $\mathrm{Ou}(x)[\operatorname{In}(y)]$ instead of $\mathrm{Ou}(\{x\})[\operatorname{In}(\{y\})]$. We will use the term bidigraph to stand for bipartite digraph. 
We will usually think of bidigraphs as being posets with the ordering $w>$ $z$ iff $w \in Y, z \in X$, and $w \in O u(z)$. It is clear in view of Definition 2 what we mean by components of $D$.

By an automorphism $F$ of $D$ we mean a bijection $F: X \cup Y \rightarrow X \cup Y$, such that $F(X)=X, F(Y)=Y$, and $(x, y) \in A$ iff $(F x, F y) \in A$.

(b) Let $X$ join-generate the lattice $L$ and $Y$ meet-generate $L$. Pick two disjoint sets $X^{\prime}, Y^{\prime}$ which have bijections $i_{1}: X \rightarrow X^{\prime}$ and $i_{2}: Y \rightarrow Y^{\prime}$. Then by the $X, Y$ bidigraph, $Q f(X, Y, L)$, of $L$ we mean the bidigraph $\left(X^{\prime}, Y^{\prime}, A\right)$ where $(x, y) \in A$ if and only if ${\overline{i_{1}}}^{-1}(x) \$ i_{2}^{-1}(y)$. Clearly $Q f(X, Y, L)$ is defined up to isomorphism. To avoid cumbersome notation we will usually suppress $i_{1}$ and $i_{2}$, and identify $X^{\prime}$ with $X$ and $Y^{\prime}$ with $Y$. In all cases where we use this convention it will be clear how to rewrite the statement in terms of $X^{\prime}, Y^{\prime}, i_{1}$, and $i_{2}$. Note that in general the group of poset automorphisms of $Q f(X, Y, L)$ is not isomorphic to the group of bidigraph automorphisms of $Q f(X, Y, L)$.

(c) Let $L$ be a complete lattice, $X$ a join-spanning subset of $L$, and $Y$ a meet-spanning subset of $L$. By $Q(X, Y, L)$ we mean the bidigraph $\left(X^{*}, Y^{*}\right.$, $A$ ) as in (b) above. We will adopt the same convention in this case concerning $X^{*}, i_{1}$, etc. In this case, the group of poset automorphisms of $Q(X, Y, L)$ is isomorphic to the group of bidigraph automorphisms of $Q(X, Y, L)$.

(d) If $L$ is a jm-lattice, by the poset of irreducibles $P(L)$ we mean $Q(J(L), M(L), L)$.

(e) Any bidigraph $(X, Y, A)$ induces a Galois connection between $2^{X}$ and $2^{Y}$ as follows. If $S \leqslant X$, then $S^{*} \leqslant Y$ is defined as $Y-\mathrm{Ou}(S)$; while if $T \leqslant Y, T^{\dagger} \leqslant X$ is defined as $X-\operatorname{In}(T)$. Note that $S^{*}$ and $T^{\dagger}$ are the sets associated with $S$ and $T$ by the usual polarities *defined on the relation $X \times Y-A$ (see [1, p. 122]).

REMARK. Note that for all $S \leqslant X, T \leqslant Y, \mathrm{Ou}\left(S^{* \dagger}\right)=\mathrm{Ou}(S)$ and $\operatorname{In}\left(T^{\dagger *}\right)$ $=\operatorname{In}(T)$. Further, note that for all $S_{1}, S_{2} \leqslant X$ (for all $\left.T_{1}, T_{2} \leqslant Y\right) \mathrm{Ou}\left(S_{1}^{*^{\dagger}}\right)$ $\leqslant \mathrm{Ou}\left(S_{2}^{* \dagger}\right)\left(\operatorname{In}\left(T_{1}^{\dagger *}\right) \leqslant \operatorname{In}\left(T_{2}^{\dagger *}\right)\right)$ iff $S_{1}^{* \dagger} \leqslant S_{2}^{* \dagger}\left(T_{1}^{\dagger *} \leqslant T_{2}^{\dagger *}\right)$, since Ou(x) $\leqslant$ $\mathrm{Ou}(S)(\operatorname{In}(y) \leqslant \operatorname{In}(T))$ iff $x \in S^{* \dagger}\left(y \in T^{\dagger *}\right)$.

The next several theorems concern the problem of reconstructing $L$ from $Q f(X, Y, L)$. It can be shown $L \cong\left\{S^{* \dagger} \mid S \leqslant X\right.$ is finite and nonempty $\}$. The same result holds if $L$ is complete, but here $S \leqslant X$ is any subset of $X$. We shall however show that $L \cong\{\mathrm{Ou}(S) \mid S \leqslant X$ is finite and nonempty $\}$. The proofs are essentially the same, but we prefer the second approach, since $\mathrm{Ou}(S)$ can be calculated more easily than $S^{* \dagger}$. Recall that we are suppressing $i_{1}, i_{2}, X^{\prime}$, and $Y^{\prime}$.

We will use the convention that whenever we write $P(L), Q(X, Y, L)$ or $Q f(X, Y, L)$ the entity in question is defined, i.e., $X, Y$ and $L$ are of the 
correct type. Thus if we write $P(L)$, we mean to imply that $L$ is a jm-lattice, etc.

THEOREM 4. Let $L$ be a lattice, $X$ join-generate $L, Y$ meet-generate $L$, and $Q f(X, Y, L)=(X, Y, \$)$.

(a) Let $R_{L}=\{\mathrm{Ou}(S) \mid S$ is a finite nonempty subset of $X\}$. The map $f: L \rightarrow R_{L}$ given by $f(a)=\mathrm{Ou}\left(X_{a}\right)=\mathrm{Ou}\left(X_{a}^{* \dagger}\right)$ is a poset isomorphism, where $X_{a}$ is as in Definition 1. Thus $R_{L}$ is a lattice isomorphic to $L$. The join of two elements in $R_{L}$ is simply their union.

(b) Each element of Aut $Q f(X, Y, L)$ (bidigraph automorphisms) extends naturally to an element of Aut $R_{L}$. More precisely, the map $F$ : Aut $Q f(X, Y, L)$ $\rightarrow$ Aut $R_{L}$ given by $F(g)(\mathrm{Ou}(S))=g(\mathrm{Ou}(S))=\mathrm{Ou}(g(S))$ is an injective group homomorphism.

(c) The image of the map $F$ above is the subgroup $\left\{\right.$ fhf $^{-1} \mid h \in$ Aut $L$ such that $h(X)=X$ and $h(Y)=Y$ (where $f$ is as in (a)) \}. Thus $F$ is a group isomorphism iff for all $h \in$ Aut $L, h(X)=X$ and $h(Y)=Y$.

Proof. Throughout the proof we will simply write $Q f$ instead of $Q f(X, Y, L)$.

(a) Observe that for all $a \in L, X_{a}^{* \dagger}=\{x \in X \mid x \leqslant a\}$. In general, for any finite nonempty $S \leqslant X, S^{* \dagger}=\left\{x \in X \mid x \leqslant \sup _{L} S\right\}$, and hence $\sup _{L} S$ $=\sup _{L} S^{* \dagger}$. Thus $a \leqslant b$ iff $f(a) \leqslant f(b)$. Thus $f$ is injective. Furthermore, $f$ is surjective since for any finite nonempty $S \leqslant X, f\left(\sup _{L} S\right)=\mathrm{Ou}\left(S^{* \dagger}\right)=$ $\mathrm{Ou}(S)$. Clearly, if $S_{1}$ and $S_{2}$ are finite and nonempty subsets of $X$, then so is $S_{1} \cup S_{2}$ and $\mathrm{Ou}\left(S_{1}\right) \cup \mathrm{Ou}\left(S_{2}\right)=\mathrm{Ou}\left(S_{1} \cup S_{2}\right)$. Thus the join of two elements in $R_{L}$ is their union.

(b) Since $g$ commutes with $\mathrm{Ou}$ and $\mathrm{In}$, it commutes with * and $\dagger$ and $F(g): R_{L} \rightarrow R_{L}$ and $F(g)$ is isotone. $F(g)$ has an isotone inverse $F\left(g^{-1}\right)$, and hence $F(g) \in$ Aut $R_{L}$. F is clearly a group homomorphism.

Suppose $F(g)=F(h)$. Then, for each $x \in X, \mathrm{Ou}(g(x))=\mathrm{Ou}(h(x))$. But $g(x)=\inf _{L}\{g(x)\}^{*}=\inf _{L}\{h(x)\}^{*}=h(x)$. Thus $g=h$ on $X$. For each $y \in$ $Y, g(y)=\sup _{L}\{g(y)\}^{\dagger}=\sup _{L} g\left(\{y\}^{\dagger}\right)=\sup _{L} h\left(\{y\}^{\dagger}\right)=\sup _{L}\{h(y)\}^{\dagger}=$ $h(y)$. Thus $g=h$ and $F$ is injective.

(c) Let $h^{\prime}=F(g)$ for $g \in$ Aut $Q f$ and $h=f^{-1} h^{\prime} f \in$ Aut $L$. Hence, $h^{\prime}=f h f^{-1}$. If $x \in X, h(x)=f^{-1} h^{\prime} f(x)=f^{-1} h^{\prime}(\mathrm{Ou}(x))=f^{-1}(\mathrm{Ou}(g(x)))=$ $g(x) \in X$. Thus $h(X)=g(X)=X$. Similarly, for $y \in Y, h(y)=f^{-1} h^{\prime} f(y)=$ $f^{-1} h^{\prime}\left(\mathrm{Ou}\{y\}^{\dagger}\right)=f^{-1}\left(\mathrm{Ou}\{g(y)\}^{\dagger}\right)=g(y)$. Thus $h(Y)=g(Y)=Y$.

Let $h \in$ Aut $L$ and suppose that $h(X)=X$ and $h(Y)=Y$. In view of our identification convention, $h \in$ Aut $Q f$. It is straightforward to verify that $F(h)=f h f^{-1}$.

REMARK. If we were to rewrite Theorem 4 in terms of the closure operator 
on $X$ (say), then part (a) would read "the map $f: L \rightarrow\left\{S^{* \dagger} \mid S \leqslant X\right.$ is finite and nonempty $\}$ given by $f(a)=X_{a}^{* \dagger}$ is a lattice isomorphism carrying meets into intersections". In part (b), the map $F$ should be given by $F(g)\left(S^{* \dagger}\right)=$ $g\left(S^{* \dagger}\right)=g(S)^{* \dagger}$. Everything else remains the same.

With very minor modifications, Theorem 4 states the properties of $Q(X, Y, L)$ $(P(L))$ for a complete lattice (jm-lattice) $L$. We shall merely state the theorems and omit the proofs, since they are almost identical to the proof of Theorem 4. For a complete lattice $L$, a join- (meet-) generating subset is a join- (meet-) spanning subset, while the converse is false. In fact, by removing 0 from any join-generating set we get a proper subset which join-spans $L$. For any jm-lattice $L$ any join- (meet-) spanning subset must contain $J(L)(M(L))$. Thus for jmlattices (especially finite lattices) $P(L)$ consists of "fewer" $(\leqslant)$ elements than $Q(X, Y, L)$ and $Q f(X, Y, L)$ (for all appropriate $X$ and $Y$ ). Note that if $L$ is a jm-lattice and $h \in$ Aut $L$, then $h(J(L))=J(L)$ and $h(M(L))=M(L)$.

THEOREM 5. Let $L$ be a complete lattice, $X$ a join-spanning subset of $L, Y$ a meet-spanning subset of $L$, and $Q(X, Y, L)=(X, Y, \nless)$.

(a) Let $\Gamma_{L}=\{\mathrm{Ou}(S) \mid S \leqslant X\}$. Then $\Gamma_{L}$ is a complete lattice (join being union) and the map $f: L \rightarrow \Gamma_{L}$ given by $f(a)=\mathrm{Ou}\left(X_{a}\right)=\mathrm{Ou}\left(X_{a}^{* \dagger}\right)$ is a lattice isomorphism.

(b) The map $F$ : Aut $Q(X, Y, L) \rightarrow$ Aut $\Gamma_{L}$ given by $F(g)(\mathrm{Ou}(S))=$ $g(\mathrm{Ou}(S))=\mathrm{Ou}(g(S))$ is an injective group homomorphism.

(c) The image of the map $F$ above is the subgroup $\left\{\right.$ fhf $^{-1} \mid h \in$ Aut $L$ such that $h(X)=X$ and $h(Y)=Y$ \}. Thus $F$ is a group isomorphism iff for all $h \in$ Aut $L, h(X)=X$ and $h(Y)=Y$.

THEOREM 6. Let $L$ be a jm-lattice and $P(L)=(J(L), M(L)$, $\$)$.

(a) Let $\Gamma_{L}=\{\mathrm{Ou}(S) \mid S \leqslant X\}$. Then $\Gamma_{L}$ is a complete lattice (join being union) and the map $f: L \rightarrow \Gamma_{L}$ given by $f(a)=\mathrm{Ou}\left(X_{a}\right)=\mathrm{Ou}\left(X_{a}^{* \dagger}\right)$ is a lattice isomorphism.

(b) The map $F$ : Aut $P(L) \rightarrow$ Aut $\Gamma_{L}$ given by $F(g)(\mathrm{Ou}(S))=g(\mathrm{Ou}(S))$ $=\mathrm{Ou}(g(S))$ is a group isomorphism.

The rest of this section is devoted to characterizing those bidigraphs which are $P(L), Q(X, Y, L)$ or $Q f(X, Y, L)$ for appropriate $L, X$, and $Y$. If $L$ is the trivial one element lattice we allow $P(L)$ and $Q(X, Y, L)$ to be $\varnothing$. The next theorem shows that any bidigraph gives rise to a complete lattice and describes a natural join-spanning set and a natural meet-spanning set.

THeOREM 7. Let $D=(X, Y, A)$ be a bidigraph, $f: X \rightarrow 2^{Y}$ be given by $f(x)=\mathrm{Ou}(x), g: \quad Y \rightarrow 2^{Y}$ be given by $g(y)=\mathrm{Ou}\left(\{y\}^{\dagger}\right)$, and $L_{D}=$ $\{\mathrm{Ou}(S) \mid S \leqslant X\}$. (When we write $\mathrm{Ou}: X \rightarrow 2^{Y}$, we mean the restriction of $\mathrm{Ou}$ 
to the subset of $2^{X}$ consisting of all singleton sets which for simplicity we identify with $X$. We treat In similarly.) Then:

(a) $L_{D}$ is a complete lattice;

(b) $f(x)-\{\varnothing\}$ join-spans $L_{D}$ and $g(Y)-\{\mathrm{Ou}(X)\}$ meet-spans $L_{D}$;

(c) there exists an injective bidigraph homomorphism $\lambda$ from $Q\left(L_{D}, f(X)-\{\varnothing\}, g(Y)-\{\mathrm{Ou}(X)\}\right)$ into $D$;

(d) $D \cong Q\left(A_{1}, A_{2}, L\right)$ iff $\lambda$ is an isomorphism. Equivalently, $D \cong$ $Q\left(A_{1}, A_{2}, L\right)$ iff Ou: $X \rightarrow 2^{Y}$ is injective, $\mathrm{Ou}(x) \neq \varnothing$ for all $x \in X$, In: $Y \rightarrow 2^{X}$ is injective, and $\operatorname{In}(y) \neq \varnothing$ for all $y \in Y$.

Proof. (a) Clearly $L_{D}$ is closed under arbitrary union and has a least element $\varnothing=\mathrm{Ou}(\varnothing)$. Hence it is a complete lattice.

(b) Clearly, $f(X)-\{\varnothing\}$ join-spans $L_{D}$. Let $S \leqslant X$. We claim that $\mathrm{Ou}(S)=\inf _{L_{D}}\left(g\left(S^{*}\right)-\{\mathrm{Ou}(X)\}\right)$. If $\mathrm{Ou}(S)=\mathrm{Ou}(X)$, this is clearly true. If $\mathrm{Ou}(S)<\mathrm{Ou}(X), \mathrm{Ou}(X) \notin g\left(S^{*}\right)$. Thus we claim that $\mathrm{Ou}(S)=\inf _{L_{D}} g\left(S^{*}\right)$. We know that $\mathrm{Ou}(S)=\mathrm{Ou}\left(S^{* \dagger}\right)$ and it is easy to see that $\mathrm{Ou}\left(S^{* \dagger}\right)=\inf _{L_{D}} g\left(S^{*}\right)$.

(c) Let $\lambda: Q\left(L_{D}, f(X)-\{\varnothing\}, g(Y)-\{\mathrm{Ou}(X)\}\right)=Q \rightarrow D$ be such that, for $a \in f(x), \lambda(a) \in f^{-1}(a)$ and, for $b \in g(Y), \lambda(b) \in g^{-1}(b)$. Clearly, $\lambda$ is injective. $\lambda$ is a bidigraph homomorphism since

$$
\begin{aligned}
& b \underset{Q}{>a} \text { iff } g(\lambda(b)) \underset{L_{D}}{\neq} f(\lambda(a)) \text { iff } \mathrm{Ou}(X-\operatorname{In}(\lambda(b))) \\
& \neq \mathrm{Ou}(\lambda(a)) \text { iff } \lambda(a) \notin X-\operatorname{In}(\lambda(b)) \text { iff } \lambda(b) \in O \mathrm{u}_{D}(\lambda(a)) .
\end{aligned}
$$

(d) If $\lambda$ is an isomorphism, then clearly $D \cong Q$. If $D \cong Q\left(A_{1}, A_{2}, L\right)$, then Ou: $X \rightarrow 2^{Y}$ is injective (thus so is $f$ ), In: $Y \rightarrow 2^{X}$ is injective (thus so is $g$ ), $\mathrm{Ou}(x) \neq \varnothing$ for all $x \in X$ (thus $\varnothing \notin f(x)$ ), and $\operatorname{In}(y) \neq \varnothing$ for all $y \in Y$ (thus $\mathrm{Ou}(X) \notin g(Y)$ ). Thus $f(X)-\{\varnothing\}=f(X)$ and $g(Y)-\{\mathrm{Ou}(X)\}$ $=g(Y)$, and since both $f$ and $g$ are injective it follows that $\lambda$ is surjective, and hence an isomorphism.

It is sometimes convenient to employ topological language to describe partially ordered sets. Thus all partial orders on a set correspond naturally in a 1-1 fashion with all $T_{0}$-topologies on the same set (see [1, p. 117]). Similarly, all quasi-orders on a set correspond in a 1-1 fashion with all topologies on the same set. We now reformulate Theorem 7 (d) in topological terms.

Corollary 8. Let $D=(X, Y, A)$ be a bidigraph. The following are equivalent:

(a) $D \cong Q\left(L, A_{1}, A_{2}\right)$.

(b) $\{\mathrm{Ou}(x)\}_{x \in X}$ is a subbase of a $T_{0}$-topology on $Y, \mathrm{Ou}(x) \neq \varnothing$ for all $y \in Y$, and In: $Y \rightarrow 2^{X}$ is injective.

(c) $\{\operatorname{In}(y)\}_{y \in Y}$ is a subbase of a $T_{0}$-topology on $X, \operatorname{In}(y) \neq \varnothing$ for all $y \in Y$, and In: $Y \rightarrow 2^{X}$ is injective. 
Proof. We will only show that (a) $\rightarrow$ (b). The rest of the proof is similar. From Theorem 7 it follows that we need only show that $\mathrm{U}_{x \in X} \mathrm{Ou}(x)=Y$, and that, for all $y_{1}, y_{2} \in Y, y_{1} \neq y_{2}$, there exists $x \in X$ such that either $y_{1} \in \mathrm{Ou}(x)$ and $y_{2} \notin \mathrm{Ou}(x)$, or $y_{1} \notin \mathrm{Ou}(x)$ and $y_{2} \in \mathrm{Ou}(x) . \mathrm{U}_{x \in X} \mathrm{Ou}(x)$ $=Y$ follows from the fact that $\operatorname{In}(y) \neq \varnothing$ for all $y \in Y$. Furthermore, if there existed $y_{1}, y_{2} \in Y, y_{1} \neq y_{2}$, such that $y_{1} \in \mathrm{Ou}(x)$ iff $y_{2} \in \mathrm{Ou}(x)$ for all $x \in X$, it would follow that $\operatorname{In}\left(y_{1}\right)=\operatorname{In}\left(y_{2}\right)$, contradicting Theorem 7 .

REMARK. If $L$ and $L^{\prime}$ are dual lattices, then it is clear that $Q\left(A_{1}, A_{2}, L\right)$ is anti-isomorphic to $Q\left(A_{1}^{\prime}, A_{2}^{\prime}, L\right)$ for appropriate $A_{1}, A_{1}^{\prime}, A_{2}, A_{2}^{\prime}$. In particular if $L$ is a jm-lattice, $L$ is self-dual iff $P(L)$ is self-dual. Conversely, if $L$ and $L^{\prime}$ are complete lattices and $g: Q\left(A_{1}, A_{2}, L\right)(=Q) \rightarrow Q\left(A_{1}^{\prime}, A_{2}^{\prime}, L^{\prime}\right)$ $\left(=Q^{\prime}\right)$ is an anti-isomorphism, then the map $f_{g}: L_{Q} \rightarrow L_{Q^{\prime}}$, given by $f_{g}(\mathrm{Ou}(\Delta))=\mathrm{Ou}\left(g(\Delta)^{\dagger}\right)$ for all $\Delta \leqslant A_{1}$ is an anti-isomorphism of lattices.

We now derive a characterization of those bidigraphs which are isomorphic to $P(L)$ for some complete jm-lattice $L$.

THEOREM 9. Let $D=(X, Y, A)$ be a bidigraph. Then the following are equivalent:

(a) $D \cong P(L)$ for some complete jm-lattice $L$.

(b) For all $x \in X$, if $\Delta \leqslant X$ is such that $\mathrm{Ou}(x)=\mathrm{Ou}(\Delta)$, then $x \in \Delta$. Similarly, for all $y \in Y$, if $\Gamma \leqslant Y$ is such that $\operatorname{In}(y)=\operatorname{In}(\Gamma)$, then $y \in \Gamma$.

Proof. If $D \cong P(L)$, then $\mathrm{Ou}(x)$ is completely join-irreducible in $L_{D}$ where join is union. Hence if $\mathrm{Ou}(x)=\mathrm{Ou}(\Delta)$, then $x \in \Delta$. Let $L^{\prime}$ be the dual of $L$, and hence a jm-lattice. Thus if $\operatorname{In}(y)=\operatorname{In}(\Gamma), y \in \Gamma$.

If (b) is true, $\mathrm{Ou}(x) \neq \varnothing$ for all $x \in X$ since $\mathrm{Ou}(\varnothing)=\varnothing$ but $x \notin \varnothing$. Further, Ou: $X \rightarrow 2^{Y}$ is injective. Similarly, $\operatorname{In}(y) \neq \varnothing$ for all $y \in Y$ and In: $Y \rightarrow 2^{X}$ is injective. Hence, by Theorem $7, D \cong Q\left(A_{1}, A_{2}, L\right)$ for a complete lattice $L$. But (b) implies that $A_{1}$ consists exactly of the completely join-irreducible elements of $L$, while $A_{2}$ consists exactly of the completely meet-irreducible elements. Thus $D \cong P(L)$.

The following theorem characterizes $Q f(X, Y, L)$. Since the proof is quite similar to the proof of Theorem 7 (one needs to be careful about the finiteness of certain sets) we will omit it.

TheOREM 10. Let $D=(X, Y, A)$ be a bidigraph. The following are equivalent:

(i) $D \cong Q f\left(A_{1}, A_{2}, L\right)$.

(ii) (a) Ou: $X \rightarrow 2^{Y}$ is injective.

(b) In: $Y \rightarrow 2^{Y}$ is injective.

(c) If $S \leqslant X$ is finite and nonempty, there exists a finite nonempty 
$T \leqslant Y$ such that $\operatorname{In}(T)=\operatorname{In}\left(S^{*}\right)$.

(d) If $T \leqslant Y$ is finite and nonempty, then there exists a finite nonempty $S \leqslant X$, such that $\mathrm{Ou}(S)=\mathrm{Ou}\left(T^{\dagger}\right)$. (Note that if $D$ is finite, then (c) and (d) imply that $\varnothing \in$ Image $\mathrm{Ou} \cap$ Image In, where $\mathrm{Ou}$ and In are restricted to finite nonempty sets.)

2. Completely distributive jm-lattices. In this section, we characterize those bidigraphs which are $P(L)$ for some completely distributive jm-lattice $L$. We begin by observing that, if $P$ is a poset, then $D(P)$ (the set of all order ideals) is a completely distributive jm-lattice, where $J(D(P))=\left\{J_{x} \mid x \in P\right\}\left(J_{x}=\right.$ $\{y \in P \mid y \leqslant x\})$ and $M(D(P))=\left\{M_{x} \mid x \in P\right\}\left(M_{x}=\{y \in P \mid y \neq x\}\right)$. Furthermore, $f: J(D(P)) \rightarrow M(D(P))$ given by $f\left(J_{x}\right)=M_{x}$ is a poset isomorphism. Conversely, if $L$ is a completely distributive jm-lattice, let $f: L \rightarrow D(J(L))$ be given by $f(x)=J_{x}$. It is easy to see that $f$ is an isomorphism of lattices. To show that $f$ is an isomorphism, we only need assume that $L$ is a complete lattice such that every element is a join of completely join-irreducibles and in which $a \wedge\left(\sup _{w \in \Delta} w\right)=\sup _{w \in \Delta}(a \wedge w)$ for all $a \in L$ and $\Delta \leqslant L$.

THEOREM 11. Let $L$ be a jm-lattice and $P(L)=(J(L), M(L), \$)$. Then the following are equivalent:

(a) $L \cong D(R)$ for some poset $R$.

(b) For all $y \in M(L), y \in \mathrm{Ou}\left(\left(M(L)-\bigcap_{x \in \operatorname{In}(y)} \mathrm{Ou}(x)\right)^{\dagger}\right)={ }_{\operatorname{def}} T_{y}$.

Proof. (a) $\rightarrow(\mathrm{b})$. For simplicity identify $L$ with $D(R)$. Let $y_{0} \in$ $M(L)$ and $x_{0} \in J(L)$ be such that $x_{0}=f^{-1}\left(y_{0}\right)$, where $f$ is the map described in the paragraph preceding this theorem. Clearly, $T_{y_{0}}=\mathrm{Ou}(S)$, where $S=$ $\left\{w \in J(L) \mid \mathrm{Ou}(W) \subset \bigcap_{x \in \operatorname{In}\left(y_{0}\right)} \mathrm{Ou}(x)\right\}$. Let $r_{0} \in R$ be such that $x_{0}=J_{r_{0}} \in$ $J(D(R))$ and $y_{0}=M_{r_{0}} \in M(D(R))$. Let $x \in \operatorname{In}\left(y_{0}\right)$; then $x=J_{r}$. Since $x \notin y_{0}$, it follows that $r \geqslant r_{0}$, i.e., $x \geqslant x_{0}$. Thus $\mathrm{Ou}(x) \geqslant \mathrm{Ou}\left(x_{0}\right)$, whence $x_{0} \in S$. But $y_{0} \in \mathrm{Ou}\left(x_{0}\right) \leqslant \mathrm{Ou}(S)=T_{y_{0}}$.

(b) $\rightarrow$ (a). Let $R=J(L)$. Let $h: L \rightarrow D(R)$ be given by $h(a)=$ $\{p \in R \mid p \leqslant a\}$. Clearly, $a \leqslant b$ iff $h(a) \leqslant h(b)$, and hence $h$ is injective.

For each $q \in M(L)$, let $u_{q} \in\left(M(L)-\bigcap_{x \in \operatorname{In}(y)} \mathrm{Ou}(x)\right)^{\dagger}$ be such that $q \in \mathrm{Ou}\left(u_{q}\right)$. Then $\mathrm{Ou}\left(u_{q}\right) \leqslant T_{q} \leqslant \mathrm{Ou}\left(u_{q}\right)$, i.e., $T_{q}=\mathrm{Ou}\left(u_{q}\right)$. Such a $u_{q}$ is unique by Theorem 9 .

Let $\phi: M(L) \rightarrow J(L)$ be given by $\phi(q)=u_{q}$. Clearly, $\phi$ is injective. Furthermore, since, for each $q \in M(L), q \in T_{q}$ it follows that for each $x \in$ $J(L), \mathrm{Ou}(x)=\mathrm{U}_{q \in \mathrm{Ou}(x)} T_{q}=\mathrm{Ou}\left(\left\{u_{q} \mid q \in \mathrm{Ou}(x)\right\}\right)$. Hence, by Theorem 9, $x=u_{q}$ for some $q \in \mathrm{Ou}(s)$. Hence $\phi$ is surjective.

Finally, let $w \in D(R)$. Then clearly $h(\sup W) \geqslant W$. Suppose $t \in h(\sup W)$. Let $q=\phi^{-1}(t), q \neq t$, and hence $q \ngtr$ sup $W$. Let $w \in W$ be such that $q \in$ 
$\mathrm{Ou}(w)$. But $\mathrm{Ou}(t)=\mathrm{Ou}\left(\left(Y-\bigcap_{x \in \operatorname{In}(q)} \mathrm{Ou}(x)\right)^{\dagger}\right) \leqslant \mathrm{Ou}(w)$, since $w \in \operatorname{In}(q)$. But $\mathrm{Ou}(t) \leqslant \mathrm{Ou}(w)$ implies that $t \in W$. Thus $h(\sup W)=W, h$ is surjective and hence an isomorphism.

3. Geometric lattices. A geometric lattice is a semimodular point lattice of finite length. In this section we characterize geometric lattices in terms of their posets of irreduribles. Although we use the same terminology used by other authors (e.g. Crapo and Rota [2]) our characterization is different. As opposed to the usual arguments, we allow our circuits to be infinite, and use induction on height instead of size. The circuits correspond to sets of the form $\operatorname{In}(y)$, as opposed to minimal dependent sets (see [2, Chapter 4]). We use the following definition.

DEFINITION 12. A matroid consists of a set $Y$ together with a family $\left\{C_{\alpha}\right\}_{\alpha \in \Delta}$ of nonempty subsets of $Y$, called circuits, such that:

(a) no proper subset of a circuit is a circuit;

(b) if $y \in C_{\alpha} \cap C_{\beta}$, then $C_{\alpha} \cup C_{\beta}-\{y\}$ contains a circuit $(\alpha \neq \beta)$.

THEOREM 13. Let $L$ be a jm-lattice, and $P(L)=(J(L), M(L), \$)$. Then the following are equivalent:

(a) $L$ is a geometric lattice.

(b) $J(L)$ together with $\{\operatorname{In}(y) \mid y \in M(L)\}$ forms a matroid and $L$ is of finite length, i.e., there exists an integer $k \geqslant 0$ such that if $T_{1}<T_{2}<T_{3}<$ $\cdots$ is a sequence of subsets of $M(L)$ for which $\operatorname{In}\left(T_{1}\right)<\operatorname{In}\left(T_{2}\right)<\cdots$, then both sequences are finite and contain no more than $k$ elements.

(c) $\mathrm{Ou}\left(x_{1}\right) \nless \mathrm{Ou}\left(x_{2}\right)$ for all $x_{1}, x_{2} \in J(L), x_{1} \neq x_{2}$ ( $L$ is a point lattice). For all $S \leqslant J(L)$, if $x_{1}, x_{2} \in J(L)$ are such that $\mathrm{Ou}(S)<\mathrm{Ou}\left(S \cup\left\{x_{1}\right\}\right) \leqslant$ $\mathrm{Ou}\left(S \cup\left\{x_{2}\right\}\right)$, then $\mathrm{Ou}\left(S \cup\left\{x_{1}\right\}\right)=\mathrm{Ou}\left(S \cup\left\{x_{2}\right\}\right)$. (L satisfies the SteinitzMacLane exchange axiom.) $L$ is of finite length, i.e., there exists an integer $k \geqslant$ 0 such that if $S_{1}<S_{2}<S_{3}<\cdots$ is a sequence of subsets of $J(L)$ for which $\mathrm{Ou}\left(S_{1}\right)<\mathrm{Ou}\left(S_{2}\right)<\cdots$, then both sequences are finite and contain no more than $k$ elements.

Proof. That (a) implies (b) follows in a straightforward manner from the standard material on geometric lattices (see [1, Chapter IV]). Using the same material one can easily see that (a) and (c) are equivalent. We now prove that (b) implies (a).

Clearly, all the elements of $M(L)$ are copoints. We now prove the following fact: If $p, q \in M(L), r, s \in J(L)$ are such that $r \in \operatorname{In}(p) \cap \operatorname{In}(q)$ and $s \in$ $\operatorname{In}(q)-\operatorname{In}(p)$, then there exists $t \in M(L)$ such that $s \in \operatorname{In}(t) \leqslant \operatorname{In}(p) \cup \operatorname{In}(q)$ $-\{r\}$. The proof we will give of this fact uses some ideas of a proof presented by Curtis Greene at the Advanced Science Seminar in Combinatorial Theory at Bowdoin 
College (Summer 1971). However, in our case we do not assume that $\operatorname{In}(p)$ is necessarily finite.

The proof is by induction on the length of the maximal chain from $p \wedge q$ to $I$. We start the induction with $d(p \wedge q, I)=2$ (where $d(a, b)=$ length of longest chain between $a$ and $b)$, since $d(p \wedge q, I)>1$. Since $J(L)$ together with $\{\operatorname{In}(y) \mid y \in M(L)\}$ form a matroid we know that there exists $t \in M(L)$ such that $\operatorname{In}(t) \leqslant \operatorname{In}(p) \cup \operatorname{In}(q)-\{r\}$. We claim that $s \in \operatorname{In}(t)$. First we show that $t \geqslant p \wedge q$. If $\lambda \in J(L)$ is such that $\lambda \leqslant p \wedge q$, then $\lambda \notin \operatorname{In}(p)$ and $\lambda \notin \operatorname{In}(q)$ and hence $\lambda \notin \operatorname{In}(t)$. Thus $t \geqslant \lambda$. But since $L$ is of finite length, $p \wedge q=\sup \{\lambda \in J(L) \mid \lambda \leqslant p \wedge q\}$, and thus $t>p \wedge q$, since $t$ is a copoint. If $s \notin \operatorname{In}(t)$ then $t \geqslant s$, and hence $t \geqslant s \vee(p \wedge q)$. But $s \notin \operatorname{In}(p)$, i.e., $p \geqslant$ $s$ and $q \neq s$. Thus $p \geqslant s \vee(p \wedge q)>p \wedge q$. But $d[p \wedge q, I]=2$ implies that $s \vee(p \wedge q)=p$ and hence that $t \geqslant p$, which is impossible since they are both copoints. Thus $s \in \operatorname{In}(t)$.

Assume the statement is true for $d[p \wedge q, I]=m \geqslant 2$ and suppose $d[p \wedge q, \eta]=m+1$. Using the property of being a matroid we see that there exists $t_{1} \in M(L)$ such that $\operatorname{In}\left(t_{1}\right) \leqslant \operatorname{In}(p) \cup \operatorname{In}(q)-\{r\}$. If $s \in \operatorname{In}\left(t_{1}\right)$, we are done. Suppose $s \notin \operatorname{In}\left(t_{1}\right)$; then $p \wedge t_{1}>p \wedge q$, since $t_{1} \geqslant p \wedge q$ (as above), $p \wedge t_{1} \geqslant s$ and $p \wedge q \neq s$. Thus $d\left[p \wedge t_{1}, I\right] \leqslant m$. Note that $r \in$ $\operatorname{In}(p)-\operatorname{In}\left(t_{1}\right)$. Since $\operatorname{In}\left(t_{1}\right) \nless \operatorname{In}(q)$, there exists $\gamma \in \operatorname{In}(t) \cap \operatorname{In}(p)-\operatorname{In}(q)$. Now by the induction hypothesis, there exists $t_{2} \in M(L)$ such that $r \in \operatorname{In}\left(t_{2}\right)$ $\leqslant \operatorname{In}(p) \cup \operatorname{In}\left(t_{1}\right)-\{\gamma\}$. Note that $s \notin \operatorname{In}\left(t_{2}\right)$ (since $s \notin \operatorname{In}(p)$ and $s \notin \operatorname{In}\left(t_{1}\right)$ by assumption). Furthermore, $q \wedge t_{2}>q \wedge p$ since $t_{2} \geqslant p \wedge t_{1}>p \wedge q$, $\gamma \leqslant q \wedge t_{2}$ and $\gamma \nless q \wedge p$. Thus $d\left[q \wedge t_{2}, I\right] \leqslant m$, and by the induction hypothesis there exists $t_{3} \in M(L)$ such that $s \in \operatorname{In}\left(t_{3}\right) \leqslant \operatorname{In}(q) \cup \operatorname{In}\left(t_{2}\right)-\{r\}$ $\leqslant \operatorname{In}(q) \cup \operatorname{In}(p)-\{r\}$, and the claim is proven.

We now show that all the elements of $J(L)$ are points. If this were false, there would exist $a, b \in J(L)$ such that $a<b$, i.e., $\mathrm{Ou}(a)<\mathrm{Ou}(b)$. Let $q \in$ $\mathrm{Ou}(a)$ and $p \in \mathrm{Ou}(b)-\mathrm{Ou}(a)$. Then $b \in \operatorname{In}(p) \cap \operatorname{In}(q)$ and $a \in \operatorname{In}(q)-\operatorname{In}(p)$. Hence by what we just proved there exists $t \in M(L)$ such that $a \in \operatorname{In}(t) \leqslant \operatorname{In}(p)$ $U \operatorname{In}(q)-\{b\}$. But this is a contradiction, since $t \in \mathrm{Ou}(a)<\mathrm{Ou}(b)$ and $b \in$ $\operatorname{In}(t)$. Thus $L$ is a point lattice.

Our next step is to show that for all $r \in J(L)$ (recall $r$ is a point), $r \notin a$ implies that $r \vee a$ covers $a$ for any $a \in L$. Suppose $r \notin a$, but $r \vee a$ does not cover $a$ for some $a \in L$. Let $b$ be such that $r \vee a>b>a$, and $b$ covers $a$. Then for some $s \in J(L), b=s \vee a$. There exist $p, q \in M(L)$ such that $p \geqslant s \vee a, p \neq r, q \geqslant a, q \geqslant r, q \neq s$. Thus $r \in \operatorname{In}(q) \cap \operatorname{In}(p)$ and $s \in \operatorname{In}(q)-\operatorname{In}(p)$. By what we proved above there exists $t \in M(L)$ such that $s \in \operatorname{In}(t) \leqslant \operatorname{In}(p) \cup \operatorname{In}(q)-\{r\}$, i.e., $t \neq s, t \geqslant r$, and $t \geqslant p \wedge q \geqslant a$. But this means that $t \geqslant r \vee a \geqslant s$, contradicting the fact that $t \ngtr s$. Hence $r \vee a$ 
covers $a$ for all $a \in L$ such that $r \notin a$.

We now show that for any $x, y \in L$, if $x$ covers $x \wedge y$, then $x \vee y$ covers $y$. Let $r \in J(L)$ be such that $x=r \vee(x \wedge y)$. Then $r \$ y$ since $x \$$ $y$. But $x \vee y=r \vee(x \wedge y) \vee y=r \vee y$, which covers $y$. Thus $L$ is (upper) semimodular by Corollary 1 of $[1$, p. 81].

Remark. Curtis Greene suggested Theorem 13 and implied that it is a known result. However, the author has not been able to find any references to it.

4. Factorization of complete lattices. Some of the following can be generalized to arbitrary lattices with universal bounds using a combination of spanning sets and generating sets. Since the results for complete lattices convey the basic idea and are more elegant, we will concentrate exclusively on complete lattices in this section.

THEOREM 14. Let $L$ be a complete lattice. Then $L \cong \Pi_{\alpha \in \Delta} N_{\alpha}\left(N_{\alpha}\right.$ a complete lattice of course) iff there exist $A_{1}, A_{2} \leqslant L$ such that $A_{1}$ joinspans $L, A_{2}$ meet-spans $L, Q\left(A_{1}, A_{2}, L\right)=\bigcup_{\alpha \in \Delta} K_{\alpha}$, where, for all $\alpha \in \Delta$, $b \in K_{\alpha} \cap A_{2}$ and $b \in \mathrm{Ou}(a) \Rightarrow a \in K_{\alpha}$ ( $K_{\alpha}$ is the union of connected components), and there exist $\left\{A_{\alpha 1}\right\}_{\alpha \in \Delta},\left\{A_{\alpha 2}\right\}_{\alpha \in \Delta}$ such that for all $\alpha \in \Delta, A_{\alpha 1}$ join-spans $N_{\alpha}, A_{\alpha 2}$ meet-spans $N_{\alpha}$, and $Q\left(N_{\alpha}, A_{\alpha 1}, A_{\alpha 2}\right) \cong K_{\alpha}$.

Proof. Necessity. $L$ is complete, so $0, I \in L$ and consequently there exist $0, I \in N_{\alpha}$ for all $\alpha \in \Delta$. Let $\left\{A_{\alpha 1}\right\}_{\alpha \in \Delta}$ and $\left\{A_{\alpha 2}\right\}_{\alpha \in \Delta}$ be such that $A_{\alpha 1}$ join-spans $N_{\alpha}$ and $A_{\alpha 2}$ meet-spans $N_{\alpha}$ for all $\alpha \in \Delta$, and let $A_{\alpha 1}^{*}=$ $\left\{x \in \Pi_{\beta \in \Delta} N_{\beta} \mid x_{\beta}=0, \beta \neq \alpha\right.$ and $\left.x_{\alpha} \in A_{\alpha 1}\right\}$. Define $A_{\alpha 2}^{*}$ similarly, but replace 0 by $I$. We will identify $L$ with the product of the $N_{\alpha}^{\prime}$ s for simplicity. It is easy to see that $A_{1}=\bigcup_{\alpha \in \Delta} A_{\alpha 1}^{*}$ join-spans $L$ and $A_{2}=\bigcup_{\alpha \in \Delta} A_{\alpha 2}^{*}$ meet-spans $L$, and that $b \in A_{2}, a \in A_{1}, b \in \mathrm{Ou}(a) \Rightarrow$ there exists $\alpha_{0} \in \Delta$ such that $b \in A_{\alpha_{0} 2}^{*}$ and $a \in A_{\alpha_{0} 1}^{*}$. From this it follows that $Q\left(A_{1}, A_{2}, L\right)$ $=\bigcup_{\alpha \in \Delta} K_{\alpha}$ with $K_{\alpha}=A_{\alpha_{2}}^{*} \cup A_{\alpha_{1}}^{*}$ and $K_{\alpha} \cong Q\left(A_{\alpha_{1}}, A_{\alpha_{2}}, N_{\alpha}\right)$.

Sufficiency. Let $\Gamma_{L}$ be as in Theorem $6, S_{\alpha}=K_{\alpha} \cap A_{2}$, and $N_{\alpha}=$ $\Gamma_{L} \cap 2^{S_{\alpha}}$ for all $\alpha \in \Delta$. Since $\Gamma_{L}$ and $2^{S_{\alpha}}$ are complete lattices where join corresponds with union, so is $N_{\alpha}$.

Consider the map $\phi: \Gamma_{L} \rightarrow \Pi_{\alpha \in \Delta} N_{\alpha}$ defined so that the $\alpha$ th component of $\phi(S)$ is $S \cap S_{\alpha}$ for $\alpha \in \Delta$, i.e., $\phi(S)=\left(S \cap S_{\alpha}\right) \alpha \in \Delta$. We claim that $\phi$ is well defined. Let $\Delta_{S} \leqslant A_{1}$ be such that $S=\mathrm{Ou}\left(\Delta_{S}\right)$. Let $W_{\alpha}=\Delta_{S} \cap K_{\alpha}$ for $\alpha \in \Delta$. Since $K_{\alpha}$ is the union of connected components, $\mathrm{Ou}\left(W_{\alpha}\right)=S \cap$ $S_{\alpha} \in N_{\alpha}$. Thus $\phi$ is well defined. $\phi$ and $\phi^{-1}$ are clearly isotone in both directions and since, for all $S \in \Gamma_{L}, S=\bigcup_{\alpha \in \Delta}\left(S \cap S_{\alpha}\right)$, it follows that $\phi$ is injective. 
Since $\Gamma_{L}$ is complete, with sup being union, it is easy to see that $\phi$ is also surjective, and hence a lattice isomorphism. Since $L \cong \Gamma_{L}$ the theorem follows.

REMARK. From Corollary 1 of $[1$, p. 68] it follows that if a complete lattice has an irreducible factorization then this factorization is unique. The following theorem shows that every jm-lattice has an irreducible representation and shows how to obtain it from $P(L)$.

THEOREM 15. Let $L$ be a jm-lattice.

(a) $L$ is decomposable iff $P(L)$ is not connected.

(b) The irreducible factors of $L$ correspond to the connected components of $P(L)$.

Proof. (a) If $P(L)$ is not connected, it follows from Theorem 14 that $L$ is decomposable. Conversely, if $L$ is decomposable it follows from Theorem 14 that there exist a join-spanning subset $A_{1}$ of $L$ and a meet-spanning subset $A_{2}$ of $L$ such that $Q\left(A_{1}, A_{2}, L\right)$ is not connected. Suppose that $P(L)$ is connected; then $P(L)$ lies in one component of $Q\left(A_{1}, A_{2}, L\right)$, call it $K$. Now either $A_{1}-K \neq \varnothing$ or $A_{2}-K \neq \varnothing$ (actually from Theorem 7 it follows that both sets are nonempty). Suppose $a \in A_{1}-K \neq \varnothing$. Then $\mathrm{Ou}(a) \cap M(L)=\varnothing$, but this means that $a \leqslant b$ for all $b \in M(L)$, i.e., $a=0$. But $0 \notin A_{1}$, by definition of join-spanning subset (the case $A_{2}-K \neq \varnothing$ is dual). This contradiction shows that $P(L)$ cannot be connected.

(b) Let the components of $P(L)$ be $\left\{K_{\alpha}\right\}_{\alpha \in \Delta}$. Applying Theorem 14 we see that $L$ is a product of the lattices corresponding to the $K_{\alpha}$ 's. Furthermore, from the first part of the proof we see that each of the factors is indecomposable since each $K_{\alpha}$ is connected. Thus we have gotten the unique irreducible factorization of $L$.

Definition 16. (a) The center of a complete lattice $L$ is the set of elements of $L$ which have one component $I$ and the other 0 , under some two factor direct factorization of $L$.

(b) An element $x \in L$, a jm-lattice, is a separator of $L$ if for all $p \in$ $J(L), q \in M(L)$ with $p \leqslant q$, either $p \leqslant x$ or $x \leqslant q$.

ReMark. Crapo and Rota [2, Corollary 1 on p. 12.11] prove that an element $x$ of a relatively complemented lattice with no infinite chains $L$ is in the center of $L$ iff $x$ is a separator of $L$. The following corollary of Theorem 15 shows that this is true for any jm-lattice. In particular, it is true for all lattices with no infinite chains.

COROLlary 17. Let $L$ be a jm-lattice and $x \in L$. Then $x \in C(L)$ (the center of $L$ ) iff $x$ is a separator of $L$.

Proof. It is clear from Theorem 15 that $x \in C(L)$ iff $B_{x} \cap K_{\alpha}=\varnothing$ or 
$B_{x} \cap K_{\alpha}=J(L) \cap K_{\alpha}$ (where $B_{x}=\{a \in J(L) \mid a \leqslant x\}$ ) for each component $K_{\alpha}$ of $P(L)(\alpha \in \Delta)$. If $p \nless q(p \in J(L), q \in M(L))$ then $p, q \in K_{\alpha_{0}}$ for some $\alpha_{0} \in \Delta$. If $B_{x} \cap K_{\alpha_{0}}=\varnothing, x \leqslant q$, whereas if $B_{x} \cap K_{\alpha} \neq \varnothing, p \in B_{x}$, i.e., $p \leqslant x$.

REMARK. Reasoning as in Corollary 17 , it is easy to see that for jm-lattice $C(L) \cong 2^{X}$, where $X$ is the set of components of $P(L)$.

\section{Examples.}

EXAMPLE 1. Let $L$ be the lattice corresponding to the following Hasse diagram:

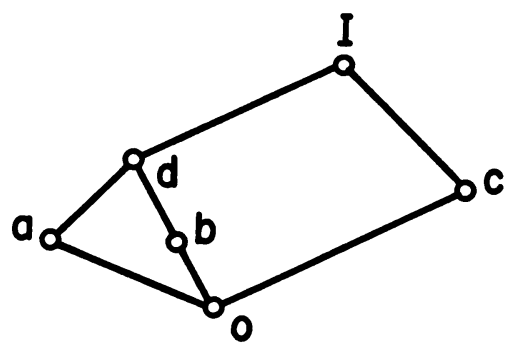

Then $P(L)$ is the following graph:

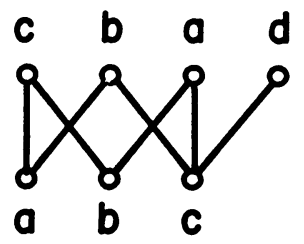

Thus $|\operatorname{Aut}(P(L))|=2=|\operatorname{Aut}(L)|$ and $L$ is indecomposable.

Finally, observe that $T_{a}=T_{b}=T_{c}=\varnothing$ and $T_{d}=\{a, b, d\}$. Thus, by Theorem 11, $L$ is not distributive, which of course is no surprise in this case. Furthermore, $Y=\{a, b, c, d\}$ together with $\{\operatorname{In}(y) \mid y \in Y\}$ is not a matroid since $\operatorname{In}(d)<\operatorname{In}(a)$. Hence, by Theorem 13, $L$ is not a geometric lattice.

EXAMPLE 2. Let $L$ be represented by the following diagram.

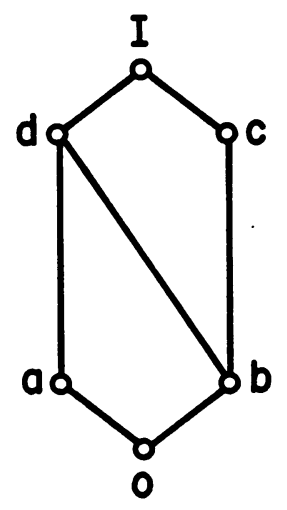


Then $P(L)$ is represented by the following diagram.
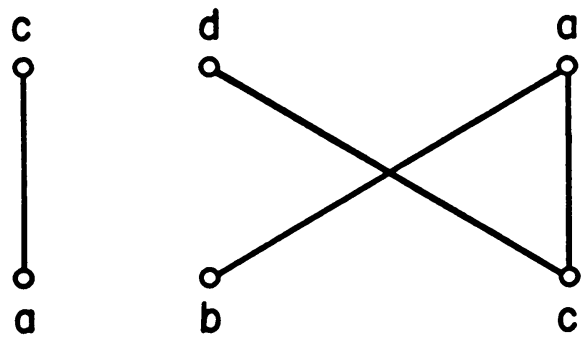

$P(L)$ has two components, so that $P(L)$ has two indecomposable factors corresponding to the diagram:
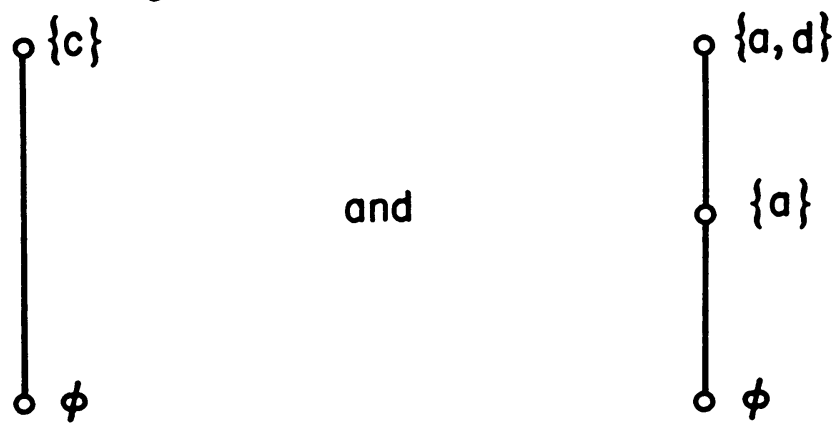

Note $|\operatorname{Aut}(P(L))|=1=|\operatorname{Aut}(L)|$. Observe that $T_{a}=\{a\}, T_{c}=\{c\}$, and $T_{d}=\{a, d\}$. Thus, by Theorem $11, L$ is distributive. By Theorem 13, we see that $L$ is not geometric, since $\operatorname{In}(d)<\operatorname{In}(a)$.

EXAMPLE 3. Let $L$ be represented by the following diagram.

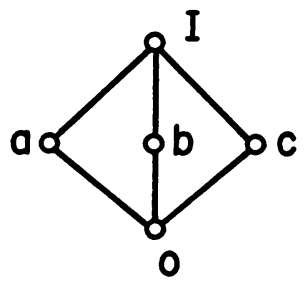

Then $P(L)$ can be represented as

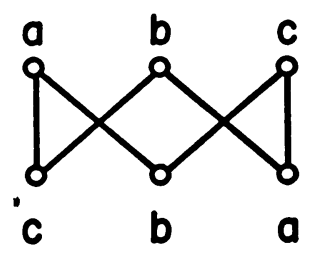

Thus $L$ is indecomposable and $|\operatorname{Aut}(P(L))|=6=|\operatorname{Aut}(L)|$. Further, note that $T_{a}=T_{b}=T_{c}=\varnothing$, and hence $L$ is not distributive by Theorem 11. The pairs $(M(L),\{\operatorname{In}(y) \mid y \in M(L)\})$ and $(J(L),\{\mathrm{Ou}(x) \mid x \in J(L)\})$ are clearly matroids and thus it follows from Theorem 13 that $L$ is a complemented modular lattice.

REMARK. Additional examples and applications of $P(L)$ can be found in [4] and [5]. The author would like to acknowledge many interesting discussions with Professors Garrett Birkhoff and Curtis Greene, as well as the many helpful suggestions they made. The author is also grateful to the referee for the time he devoted to increasing the clarity of this paper. 


\section{BIBLIOGRAPHY}

1. G. Birkhoff, Lattice theory, 3rd ed., Amer. Math. Soc. Colloq. Publ., vol. 25, Amer. Math. Soc., Providence, R. I., 1967. MR 37 \#2638.

2. H. H. Crapo and G.-C. Rota, On the foundations of combinatorial theory: II. Combinatorial geometries, (preliminary edition), M. I. T. Press, Cambridge, Mass., 1970.

3. P. Crawley and R. P. Dilworth, Algebraic theory of lattices, Prentice-Hall, Englewood Cliffs, N. J., 1973.

4. G. Markowsky, Some combinatorial aspects of lattice theory. Proc. Houston Lattice Theory Conf. , Univ. of Houston Press, 1973, pp. 36-38.

5. Combinatorial aspects of lattice theory with applications to the enumeration of free distributive lattices, Ph. D. Thesis, Harvard University, Cambridge, Mass., June 1973.

\section{DEPARTMENT OF MATHEMATICS, HARVARD UNIVERSITY, CAMBRIDGE, MASSACHUSETTS 02138}

Current adress: Computer Science Department, IBM T. J. Watson Research Center, P. O. Box 218, Yorktown Heights, New York 10598 